NOTICE: this is the author's version of a work that was accepted for publication in Computers and Chemical Engineering. Changes resulting from the publishing process, such as peer review, editing, corrections, structural formatting, and other quality control mechanisms may not be reflected in this document. Changes may have been made to this work since it was submitted for publication. A definitive version was subsequently published in Computers and Chemical Engineering, Vol. 58 (2013).

DOI: 10.1016/j.compchemeng.2013.07.012 


\title{
Continuous prediction technique for fast determination of cyclic steady state in simulated moving bed process
}

\author{
Chuanyi Yao ${ }^{\mathrm{a},}{ }^{*}$, Shaokun Tang ${ }^{\mathrm{b}}$, Hong-Mei Yao ${ }^{\mathrm{c}}$, Moses O. Tade ${ }^{\mathrm{c}}$ \\ ${ }^{a}$ Department of Chemical and Biochemical Engineering, College of Chemistry and Chemical Engineering, \\ Xiamen University, Xiamen, Fujian, 361005, China \\ ${ }^{\mathrm{b}}$ College of Chemical Engineering, Tianjin University, Tianjin, 300072, China \\ ${ }^{\mathrm{C}}$ Centre for Process Systems Computations, Department of Chemical Engineering, Curtin University, GPO \\ Box U1987, Perth, WA 6845, Australia
}

*Corresponding author. Tel.:+86-592-2181360.

E-mail address: cyao@xmu.edu.cn (C. Yao).

Keywords: Simulated moving bed, Cyclic steady state, Continuous predicting method, Acceleration, Adsorptive separation

\begin{abstract}
In the simulation of cyclic processes, such as simulated moving bed (SMB), the system should be equilibrated to reach a cyclic steady state (CSS) before evaluating the process performance. However, the conventional method of successive substitution is quite time-consuming. In this work, a continuous predicting method (CPM) is developed for fast determination of CSS in SMB. In CPM, the direct prediction of state variable at CSS and solving model equation are conducted alternately until CSS is reached. In order to give a guideline for the selection of the acceleration factor, CPM is applied on SMB process for enantioseparation of 1,1'-bi-2-naphtol racemate and with the optimized acceleration factor, $59 \%$ of computation time saved compared with successive substitution. In addition, this method is further successfully used in a sugar separation process. Given its efficiency and simplicity, this method could provide a useful tool for SMB simulations.
\end{abstract}




\section{Introduction}

Cyclic processes are operations executed cycle by cycle. The stationary regime of this kind of processes is a cyclic steady-state (CSS), in which the state at the certain time of a switch period is identical to the state at that time instant of the last switch period, while the system states are still varying over time. Due to its high productivity, cyclic operation has been applied to many processes, cyclic reactor, cyclic separator, cyclic combustion, cyclic wetting and drying, cyclic latent heat thermal storage etc.

As one of the cyclic processes, simulated moving bed (SMB) chromatographic process is powerful in the separation of chemically similar components (Rajendran et al., 2009; Gomes and Rodrigues, 2012). In SMB, the continuous and countercurrent movement of liquid and solid phase is realized by the periodical switching of inlet and outlet ports in the direction of liquid phase flow (Minceva el al., 2003), the solid phase is not actually moved. Compared with the classical batch preparative chromatography, the SMB process has the advantages of enhanced mass transfer driving force, efficient use of packing material and desorbent, high productivity, and less dilution of products. Therefore SMB technology has been applied in many fields, including petroleum (Broughton, 1984; Lim, 2012), food (Ching \& Ruthven, 1985; Beste et al., 2000), biotechnology (Horneman et al. 2007; Andersson et al. 2008; Li et al. 2007; Gueorguieva et al., 2011), pharmaceutical and fine chemical industries (Negawa \& Shoji, 1992; Pais et al. 1998).

In the early stage of design, numerical simulation is a vital and quick tool to evaluate the performance at different operation conditions quantitatively. However, the process performance is evaluated only after the system reaches CSS. Thus, the determination of CSS from dynamic modeling becomes important.

Generally, SMB model is set up by the mass balance-equation in both liquid and solid phases, which could be solved to obtain the state variable (concentrations in the liquid and solid phases). The state variable is the function of time, position and cycles. When CSS is reached, the state variable is independent of cycles, therefore, the state variable in the model only depend on the time and position. Usually, the model equations are solved by numerical methods and iteration is needed. The 
initial state variable (initial condition) is used to solve the model equation and a new state variable could be obtained, which is used as the initial condition for the next iteration. When the two neighboring state variables are close to a specific extent (depending on the requirement), we take it as reaching convergence and the last profile of state variable describe the state at CSS. Traditionally, the solution process mimics the actual physical process to reach CSS, i.e. one iteration is corresponding to one switch in the system operation. While the actual system may take hundreds of switches, with this conventional method, so large amount of iteration time is needed, and hence it is computationally inefficient.

In the past few years, several works have been done in order to accelerate the calculation of the periodic steady state. In the SMB process, some more effective numerical solvers were proposed, such as, the space time conservation element and solution element method (Lim and Jorgensen, 2004; Lim et al. 2004), and cascadic multilevel approach (Lubke et al. 2007). Another way of acceleration is to predict CSS directly, which is based on the fact that initial conditions do not affect the state at CSS (Minceva et al., 2003; Hidajat et al., 1986). Nevertheless, the initial conditions have impact on the switch numbers needed to reach CSS, shown by Hidajat and his coworkers (Hidajat et al., 1986). They found that the number of switches to achieve CSS is reduced if some columns are initialized with a certain concentration of solutes. Therefore, it is desirable to choose an appropriate initial condition for accelerating simulation of SMB. However, it is not easy since the pre-set state is far from CSS, shown in the work of Minceva et al. with non-void initial columns (Minceva et al., 2003). Certainly, the close is the initial state to that at CSS, the lesser switch number is needed to achieve CSS, but the state at CSS is unknown before reaching it. Recently, a new method based on the concept of "quasi-envelope" was used to predict the CSS via a pseudo-smooth function (Yao et al., 2010). But it encounters the difficulties in determining the step size and the time to activate the quasi-envelope computation.

In view of the wide application of cyclic operation and the intensive computation involved in optimization design, this research aims to provide an efficient simulation strategy independent of initial conditions. In this work, a continuous predicting method (CPM) is developed for fast 
determination of the CSS. The scheme stems from the fact that for SMB operation, stationary states are determined by the system structure and operating parameters, not the column initial condition.

\section{Mathematical modeling of SMB process}

A SMB model consists of a single chromatographic column model and several equations for the mass balance at the nodes of the system, Which connect columns together according to the specific configurations. Column models have been reviewed in many literatures (Yao et al., 2008; Guiochon, 2002). The representing models include general rate (Dunnebier et al., 2000), linear driving force (LDF) (Yao et al., 2008; Yao et al., 2010), transport-dispersive equilibrium (Lubke et al., 2007), and ideal model (Dunnebier and Klatt, 2000; Zhong and Guiochon, 1996). Among them, LDF model was proved to be a good compromise between accuracy and simplicity (Biegler et al., 2004). Therefore, LDF is used in this work to describe the kinetics of each column.

The mass balance equations for component $i$ in the bulk flow phase and in the solid phase are as follows:

$\frac{\partial c_{i}}{\partial t}+v \frac{\partial c_{i}}{\partial x}=D_{\mathrm{a}, i} \frac{\partial^{2} c_{i}}{\partial x^{2}}-\frac{1-\varepsilon_{\mathrm{b}}}{\varepsilon_{\mathrm{b}}} k_{\mathrm{e}, i}\left(q_{i}^{*}-q_{i}\right)$

$\frac{\partial q_{i}}{\partial t}=k_{\mathrm{e}, i}\left(q_{i}^{*}-q_{i}\right)$

where $q_{i}^{*}$ is governed by a specific adsorption isotherm:

$q_{i}^{*}=g_{i}\left(c_{\mathrm{A}}, c_{\mathrm{B}}\right)$

To complete the dynamic model system, initial conditions and boundary conditions are needed. The initial conditions describe the status at the beginning of a switching period:

$c_{i}^{(k)}(0, x)=c_{i}^{(k-1)}\left(t_{\mathrm{s}}, x\right)$

$q_{i}^{(k)}(0, x)=q_{i}^{(k-1)}\left(t_{\mathrm{s}}, x\right)$

where $k$ is the number of switching. If the SMB unit starts from clean columns, then:

$c_{i}^{(1)}(0, x)=q_{i}^{(1)}(0, x)=0$.

Boundary conditions are needed for both ends of each column: 


$$
\begin{aligned}
& \left.\frac{\partial c_{i}}{\partial x}\right|_{x=0}=\frac{v}{D_{\mathrm{a}, i}}\left(c_{i}-c_{i}^{\mathrm{in}}\right) \\
& \left.\frac{\partial c_{i}}{\partial x}\right|_{x=L}=0
\end{aligned}
$$

Here, $c_{i}^{\text {in }}$ is subject to the mass balances at the nodes described as follows.

Desorbent node:

$c_{i, \mathrm{I}}^{\mathrm{in}} Q_{\mathrm{I}}=c_{i, \mathrm{IV}}^{\mathrm{out}} Q_{\mathrm{IV}}+c_{i, \mathrm{D}} Q_{\mathrm{D}}$

$Q_{\mathrm{I}}=Q_{\mathrm{IV}}+Q_{\mathrm{D}}$

Extract node:

$c_{i, \mathrm{II}}^{\text {in }}=c_{i, \mathrm{I}}^{\mathrm{out}}=c_{i, \mathrm{E}}$

$Q_{\mathrm{II}}=Q_{\mathrm{I}}-Q_{\mathrm{E}}$

Feed node:

$c_{i, \mathrm{III}}^{\text {in }} Q_{\mathrm{III}}=c_{i, \mathrm{II}}^{\mathrm{out}} Q_{\mathrm{II}}+c_{i, \mathrm{~F}} Q_{\mathrm{F}}$

$Q_{\mathrm{III}}=Q_{\mathrm{II}}+Q_{\mathrm{F}}$

Raffinate node:

$c_{i, \mathrm{IV}}^{\text {in }}=c_{i, \mathrm{III}}^{\text {out }}=c_{i, \mathrm{R}}$

$Q_{\mathrm{IV}}=Q_{\mathrm{III}}-Q_{\mathrm{R}}$

The Eqs.(1)-(14) construct a partial differential algebraic equation (PDAE) system. For simplicity, we define a vector of state variable:

$\boldsymbol{u}=\left[\begin{array}{llll}c_{\mathrm{A}} & c_{\mathrm{B}} & q_{\mathrm{A}} & q_{\mathrm{B}}\end{array}\right]$

The state variable, vector $\boldsymbol{u}$, is function of time and axis position. For solving the SMB model equations, the discretization of spatial derivatives is conducted. Then the resulted ordinary differential equations are integrated in time (by function ODE45 in MATLAB) using method of lines (Lim et al., 2004; Yao et al., 2008). The protocol is shown in Fig.1 and described as follows.

First, all the columns are initialized with $\boldsymbol{u}=0$ (for void column). Then, the model equations, Eq.(1) and Eq.(2), are solved to get $\boldsymbol{u}$ at $t=t_{s}$. In this process, $q_{i}^{*}$ is obtained through a system-dependent isotherm, Eq.(3). In the boundary condition, Eq.(5), $c_{i}^{\text {in }}$ can be calculated through Eq.(7)-(14) depending on column number, for example, $c_{i}^{\text {in }}$ for the first column in zone I (i.e. column 1 ) is obtained by solving Eq.(7) and Eq.(8). Before solving $\boldsymbol{u}$ at any time level $t$, all the values of $c_{i}^{\text {in }}$ 


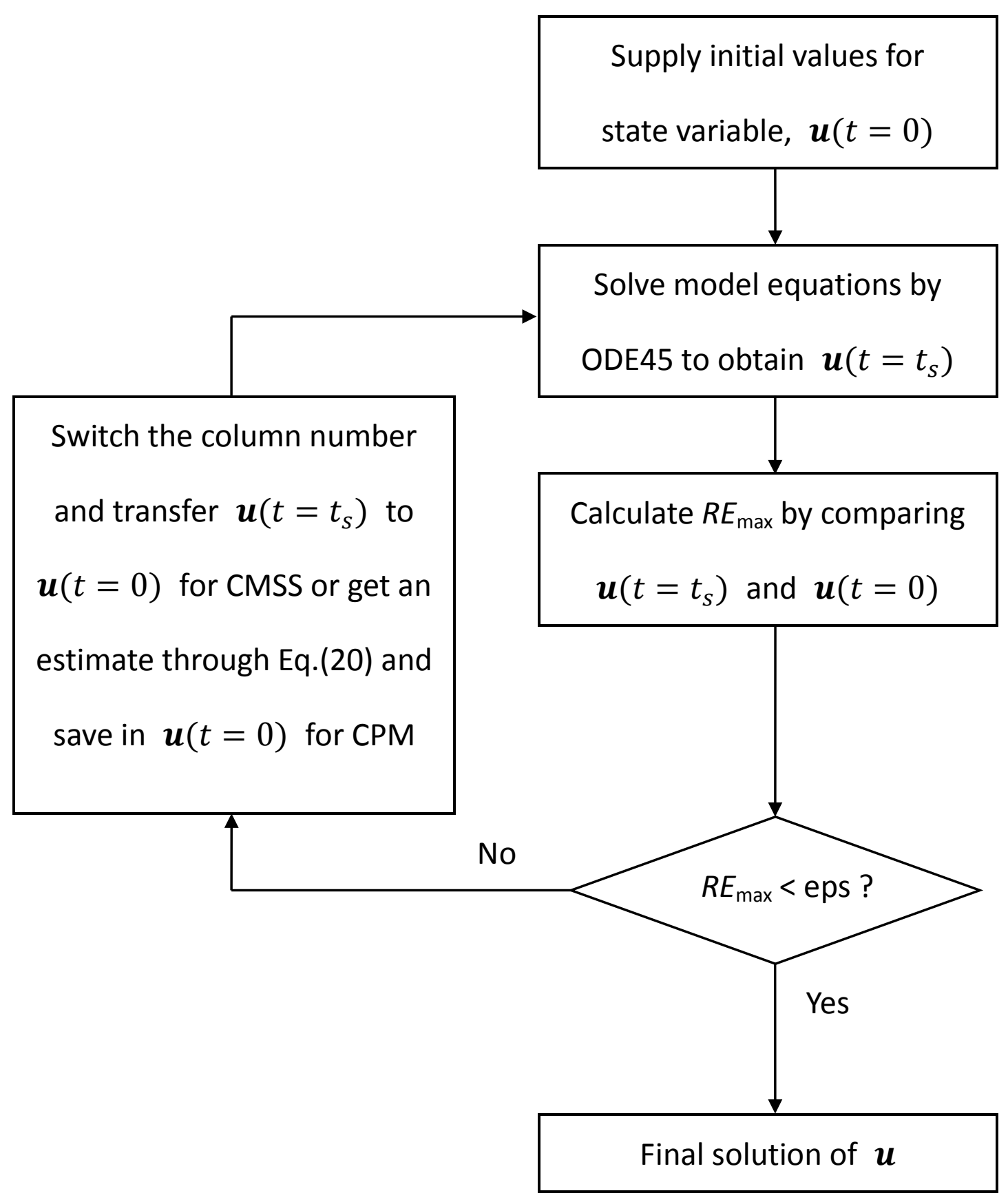

Fig. 1. Protocols of conventional method (CMSS) and continuous predicting method (CPM).

are obtained through $c_{i}^{\text {out }}$ at the last time level, $t-\Delta t$, which are consecutively updated with time in the solution process. For convergence, at each iteration, the difference between $\boldsymbol{u}\left(t=t_{s}\right)$ and $\boldsymbol{u}(t=0)$ (which is $\boldsymbol{u}\left(t=t_{s}\right)$ for the last switch period) is calculated and compared to the criterion 
for achieving CSS. If the criterion is not fulfilled, after switching the column number (plus 1 for column 1-7 and set 1 for column 8$)$ and setting $\boldsymbol{u}\left(t=t_{s}\right)$ as initial value for the next switch, the model equation is solved again. This process is repeated until reaching CSS.

The criterion for achieving CSS is defined as the difference of state variable at the end of consecutive switches. Here, the relative errors $(R E)$ of the average concentrations of each component in the extract and raffinate streams are defined in Equation (16) (Minceva et al., 2003):

$R E_{i, \mathrm{E} / \mathrm{R}}=\left|\frac{\bar{c}_{i, \mathrm{E} / \mathrm{R}}^{(k)}-\bar{c}_{i, \mathrm{E} / \mathrm{R}}^{(k-1)}}{\bar{c}_{i, \mathrm{E} / \mathrm{R}}^{(k)}}\right|$

where the average concentration is calculated by Equation (17):

$\bar{c}_{i, \mathrm{E} / \mathrm{R}}^{(k)}=\frac{1}{t_{\mathrm{s}}} \int_{(k-1) t_{\mathrm{s}}}^{k t_{\mathrm{s}}} c_{i, \mathrm{E} / \mathrm{R}}(t) d t$

Then, the maximum value $\left(R E_{\max }\right)$ among the four relative errors $\left(R E_{\mathrm{A}, \mathrm{E}}, R E_{\mathrm{B}, \mathrm{E}}, R E_{\mathrm{A}, \mathrm{R}}, R E_{\mathrm{B}, \mathrm{R}}\right)$ is used as the criterion of achieving CSS:

$R E_{\max }<$ eps

where eps is a small value. We used $10^{-6}$ in this work.

\section{Continuous predicting method (CPM)}

In the conventional method, the system model needs to run as many times as switches in real operation. A typical time profile of the concentration distribution along the column length is depicted in Fig. 2. With eps of $10^{-6}$, it takes 156 switches to reach CSS.

In most of the simulation work, such as the optimization of operation conditions using numerical method, only the state variables at CSS are taken into account. In order to check if the initial conditions influence the final CSS state, different initial concentrations in fluid phase (while the initial concentrations in solid phase are set to be equilibrium with the fluid phase) are used (Fig.3),. As a result, the system tends to approach the same CSS.

Unlike conventional method, where the end state of last switch is used as the initial value for the next switch, the method proposed here is free to choose an estimated CSS as the initial condition. 

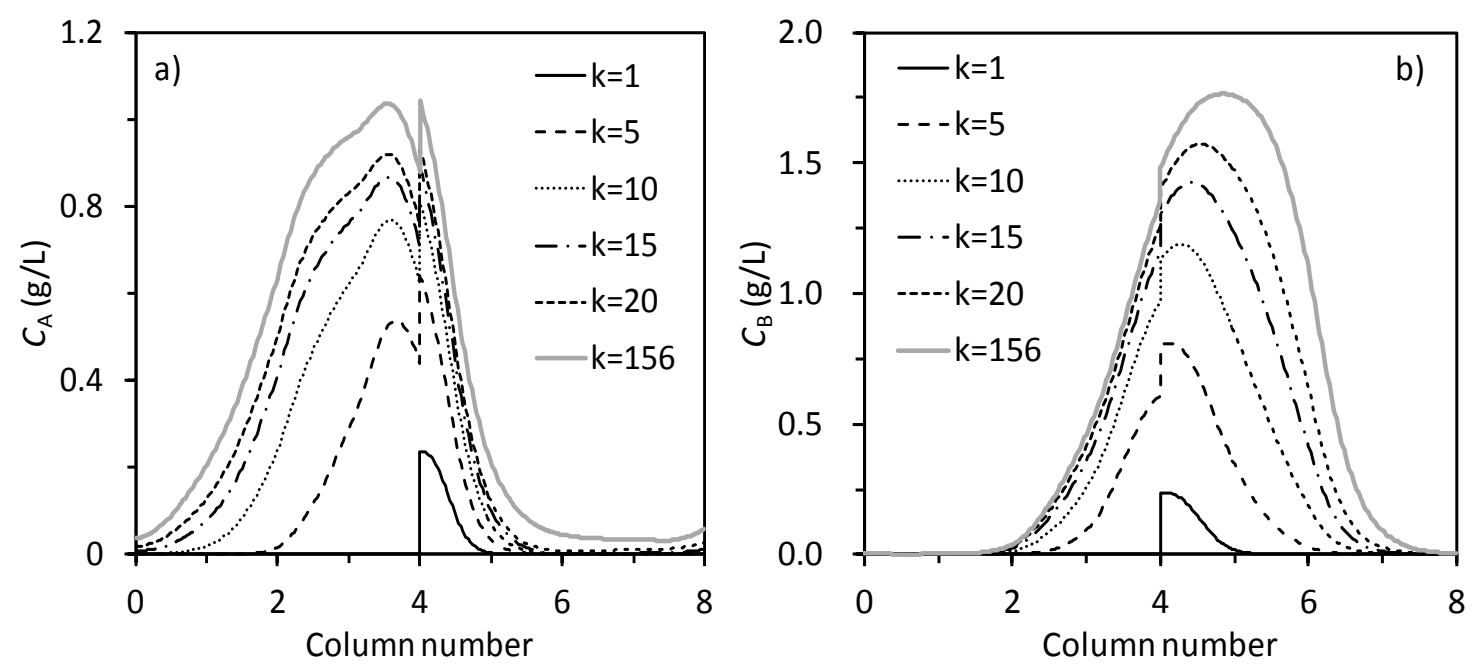

Fig. 2. Propagation of internal concentration profiles for enantiomers separation.

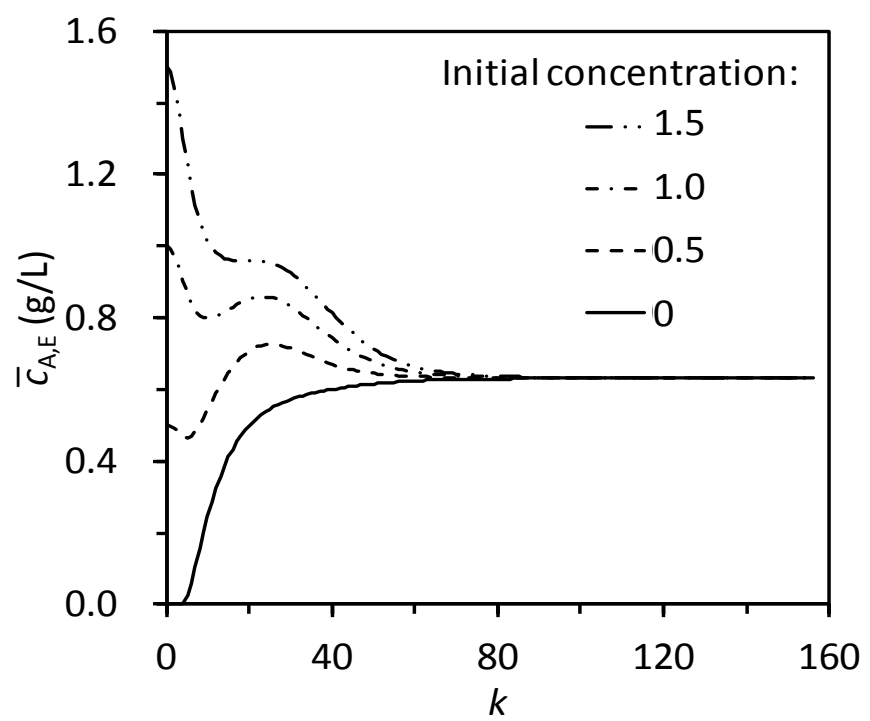

Fig. 3. Time profile of concentration at particular axial point (extract stream) starting from different initial conditions.

However, our method does not rely on a good initial condition. Instead, we found another way to accelerate the iteration with an acceleration factor which controls the convergence efficiency. As you can see in Fig.2, at each iteration, the state variable, $\boldsymbol{u}^{(k)}$, is approaching the variable at CSS, $\boldsymbol{u}_{\text {CSS }}$. Notably, the difference of $\boldsymbol{u}^{(k)}-\boldsymbol{u}^{(k-1)}$, is also decreasing with $k$, same as $\boldsymbol{u}_{\mathrm{CSS}}-\boldsymbol{u}^{(k)}$. Then they could be correlated like this: 


$$
\boldsymbol{u}_{\mathrm{CSS}}-\boldsymbol{u}^{(k)}=\theta\left[\boldsymbol{u}^{(k)}-\boldsymbol{u}^{(k-1)}\right]
$$

With this correlation, we proposed a general function for the prediction of $\boldsymbol{u}_{\mathrm{CSS}}$ :

$\boldsymbol{u}_{\mathrm{CSS}}^{(n)}=\boldsymbol{u}^{(n)}+\theta\left[\boldsymbol{u}^{(n)}-\boldsymbol{u}_{\mathrm{CSS}}^{(n-1)}\right]$

where $\boldsymbol{u}_{\mathrm{CSS}}^{(n)}$ is the prediction after the $n$th iteration, $\boldsymbol{u}^{(n)}$ is the solution of the model equation with $\boldsymbol{u}_{\mathrm{CSS}}^{(n-1)}$ as the initial conditions, and $\theta$ is the acceleration factor. Let $\boldsymbol{u}_{\mathrm{CSS}}^{(1)}=\boldsymbol{u}^{(1)}$, as an initial estimate.

To evaluate if the prediction from Eq.(20) could bring out the state of CSS, $\boldsymbol{u}_{\text {CSS }}^{(n)}$ is used as the initial state of the SMB model and the PDAEs are solved for one switching period to get $\boldsymbol{u}^{(n+1)}$. The resulted state variable is compared with $\boldsymbol{u}_{\mathrm{CSS}}^{(\boldsymbol{n})}$, and the relative error is calculated. The scheme of this method is shown in Fig. 4b.

a) Conventional method

$$
\boldsymbol{u}^{(1)} \stackrel{\mathrm{PDAE}}{\longrightarrow} \boldsymbol{u}^{(2)} \stackrel{\mathrm{PDAE}}{\longrightarrow} \boldsymbol{u}^{(3)} \cdots \boldsymbol{u}_{\mathrm{CSS}}
$$

b) CPM

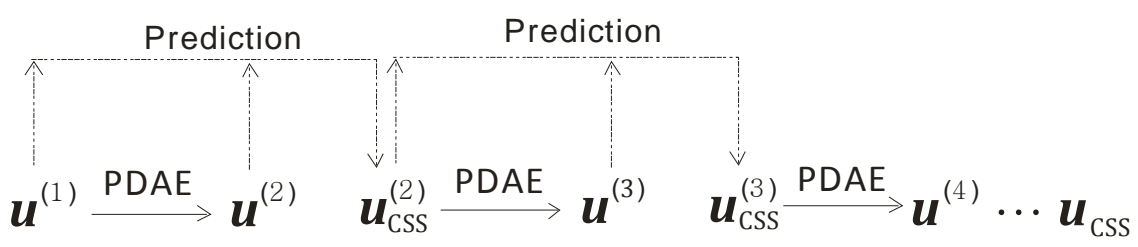

Fig. 4. Calculation scheme of conventional method and CPM

To sum up, the process includes the following steps:

Step 1. The initial condition, $\boldsymbol{u}^{(1)}$, is used to solve PDAE in the same way as conventional method (Fig. 4a) to generate $\boldsymbol{u}^{(2)}$, and, set $\boldsymbol{u}_{\text {CSS }}^{(1)}=\boldsymbol{u}^{(1)}$;

Step 2, $\boldsymbol{u}_{\text {CSS }}^{(1)}$ and $\boldsymbol{u}^{(2)}$ are used to predict a new estimate of the state at CSS, $\boldsymbol{u}_{\text {CSS }}^{(2)}$, through Eq. 
(20);

Step 3, $\boldsymbol{u}_{\text {CSS }}^{(2)}$ is used as the initial condition for system model PDAE to generate $\boldsymbol{u}^{(3)}$. The relative error between $\boldsymbol{u}_{\text {CSS }}^{(2)}$ and $\boldsymbol{u}^{(3)}$ is calculated and the criterion of CSS is examined.

Step 2 and Step 3 are repeated until the steady state is achieved.

It is obvious that CPM does iteration, same as the conventional method, which brings similar protocols (see Fig.1). However, their iteration ways are different. In CMSS, the state variable at the end of one iteration, i.e. $\boldsymbol{u}\left(t=t_{s}\right)$, is used as the initial conditions for the next iteration, while in CPM, the initial conditions of each iteration $(n>2)$ is obtained through Eq.(20). If $\theta=0$, CPM is restored to CMSS.

In the following sections, the above accelerating method is applied to SMB separation processes to demonstrate its capability of fast simulation in determining CSS.

\section{Application of CPM to SMB cyclic operation}

\subsection{Choosing an acceleration factor}

In this continuous predicting method, choosing a proper acceleration factor $\theta$ is the key to speed up reaching CSS. Here we take the separation of racemic 1,1'-bi-2-naphtol (Pais et al., 1998) as an example to investigate the effect of $\theta$ on the CPM performance. The operation conditions and model parameters used are adopted from reference (Pais et al., 1998) and listed in Table 1.

Because the computational time is proportional to the iteration number (Fig.5), the CPM performance is described by the iteration number. The iterations required to achieve CSS with different acceleration factors are presented in Fig.5. It shows that the required iteration number is reduced with the increasing of $\theta$ in the range of $[0,0.5]$. If $\theta>0.5$, the iteration number is increasing and reaches 165 at $\theta=0.7$, even more than that of CMSS (156). Additionally, the curve takes the bell-shape, implying that the iteration number reaches a minimum with $\theta$ of 0.5 , where only 64 iterations are needed, much less than 156 from CMSS.

Since the criterion for reaching CSS is eps dependent, the influence of $\theta$ on CPM performance at different eps is also conducted, shown in Fig. 6 . Although the iteration numbers of both CMSS (i.e. 
Table 1. Parameters for enantioseparation of 1,1'-bi-2-naphthol

\begin{tabular}{ll}
\hline Items & Values \\
\hline Configuration & $2 / 2 / 2 / 2$ \\
Column & i.d. $2.6 \mathrm{~cm} \times 10.5 \mathrm{~cm}$ \\
Porosity & $\varepsilon_{\mathrm{b}}=0.4$ \\
Dispersion coefficient, $\mathrm{cm}^{2} / \mathrm{min}^{-1}$ & $D_{\mathrm{a}}=10.5 \times 10^{-3} \mathrm{v}$ \\
Mass transfer coefficient, $\mathrm{min}^{-1}$ & $k_{\mathrm{e}}=6.0$ \\
Feed concentration, $\mathrm{g} / \mathrm{L}$ & $c_{\mathrm{A}}=2.9, c_{\mathrm{B}}=2.9$ \\
Switching time, min & $t_{\mathrm{s}}=2.75$ \\
Flow rate, $\mathrm{mL} / \mathrm{min}$ & $Q_{\mathrm{I}}=56.83, Q_{\mathrm{II}}=40.83, Q_{\mathrm{III}}=44.47, Q_{\mathrm{IV}}=35.38$ \\
& $q_{\mathrm{A}}^{*}=\frac{3.73 c_{\mathrm{A}}}{1+0.0466 c_{\mathrm{A}}+0.0336 c_{\mathrm{B}}}+\frac{0.3 c_{\mathrm{A}}}{1+3 c_{\mathrm{A}}+c_{\mathrm{B}}}$ \\
Equilibrium isotherm & $q_{\mathrm{B}}^{*}=\frac{2.69 c_{\mathrm{B}}}{1+0.0466 c_{\mathrm{A}}+0.0336 c_{\mathrm{B}}}+\frac{0.1 c_{\mathrm{B}}}{1+3 c_{\mathrm{A}}+c_{\mathrm{B}}}$ \\
\hline
\end{tabular}

$\theta=0$ ) and CPM decrease with the increasing eps, at eps of $10^{-6}$ (Fig.5), $10^{-5}$ and $10^{-4}$ (Fig.6), the optimum $\theta$ are all 0.5 . This indicates that the optimum $\theta$ is not sensitive to eps.

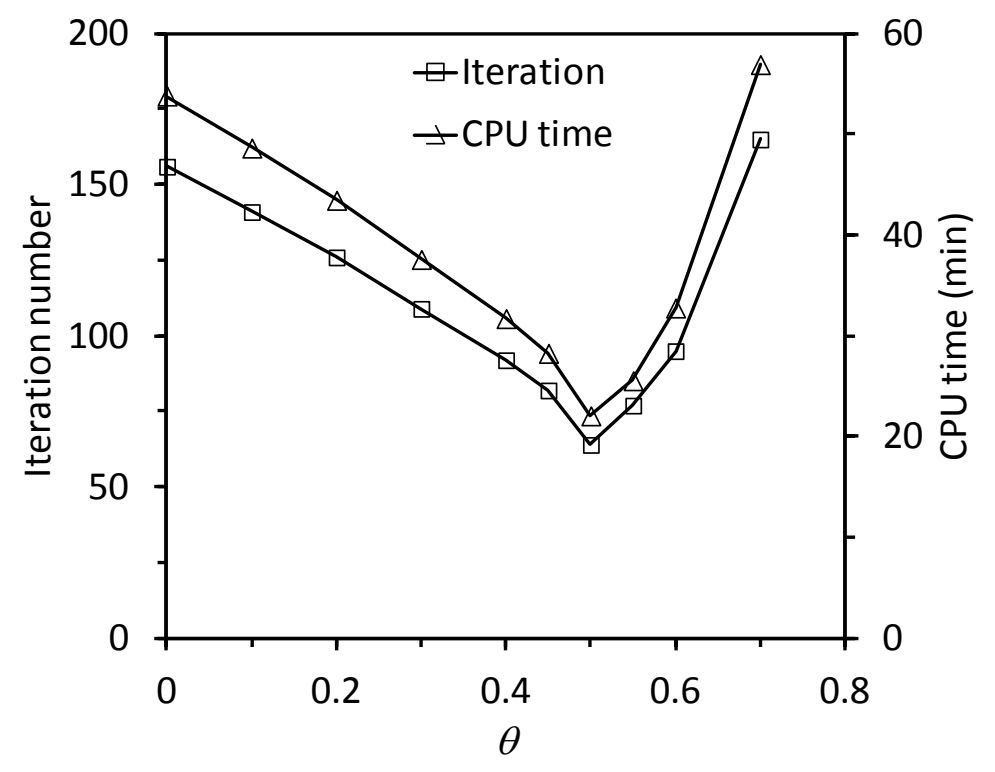

Fig.5. Iteration number and computational time needed for achieving CSS in SMB process of enantioseparation of racemic 1,1'-bi-2-naphthol by CPM. 


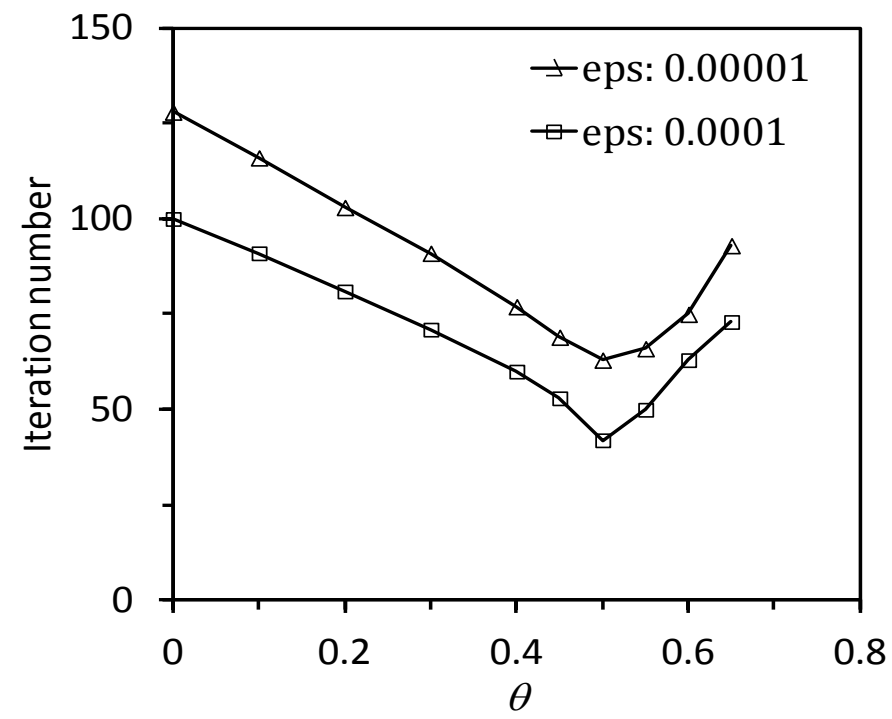

Fig.6. Effect of eps on the iteration numbers needed for achieving CSS in SMB process of enantioseparation of racemic 1,1'-bi-2-naphthol by CPM.

To characterize the state each iteration reaches, concentrations in the extract and raffinate streams were monitored and their convergences are shown in Fig. 7. Compared with CMSS, the concentration curves calculated by CPM approach the values at CSS more rapidly, consistent with the smaller iteration numbers.
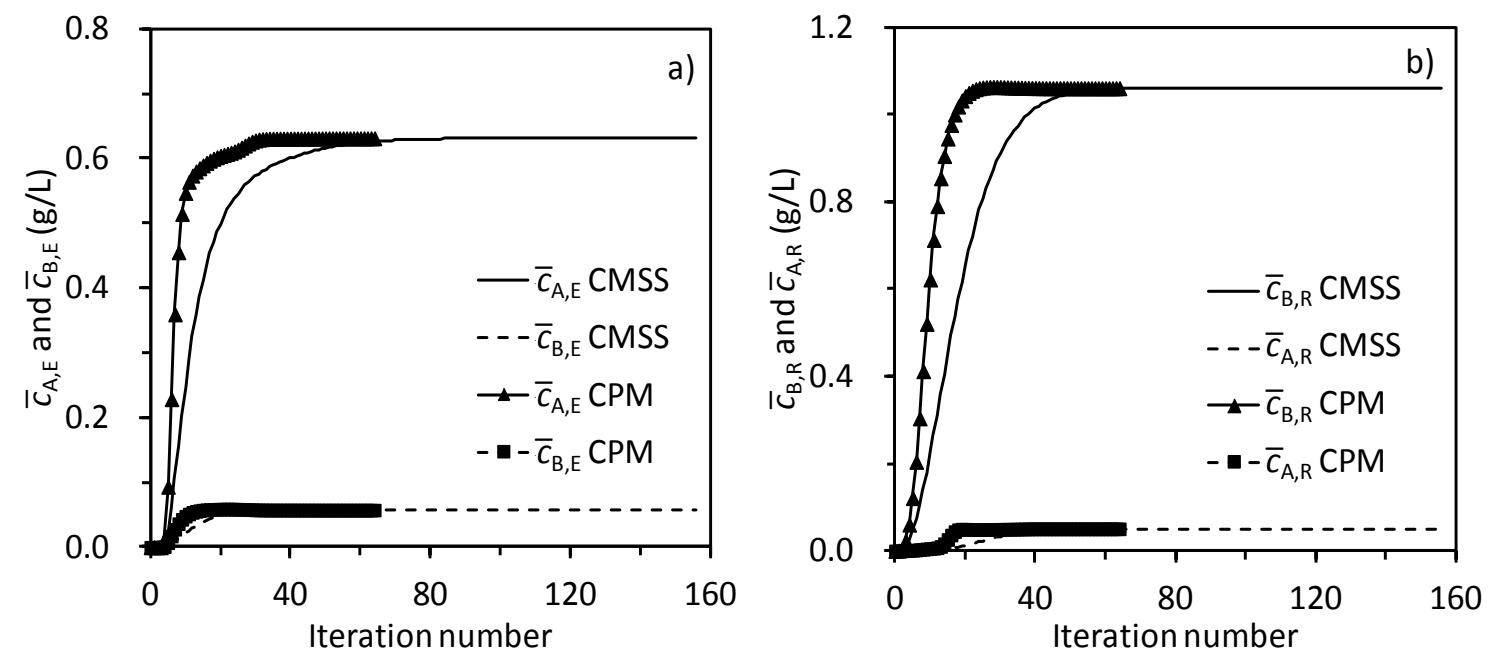

Fig.7. Profile of convergence to CSS concentration in the extract (a) and raffinate (b) streams in SMB process for enantioseparation of 1,1'-bi-2-naphtol calculated by CMSS and CPM with $\theta=0.5$. 
Notably, the concentration curves calculated by CPM are different from the curves obtained in CMSS. As we know, the solution from CMSS method models the entire process of real system. Any point in the concentration curves obtained by CMSS is corresponding to a real operation state. However, CPM method does not record real states. Some values in $\bar{c}_{\mathrm{B}, \mathrm{R}}$ curve calculated by CPM (Fig. 7b) are even higher than the value at CSS, while in the real operation, the average concentration of less retained compound in raffinate is always lower than the value at CSS. Besides, similar to the real state, the value of $n$ does not refer to the switch number in real system either. Therefore, CPM can only be used when the transient is not of interest.

In different separation systems, the optimal $\theta$ may be different. However, $\theta=0.5$ can be chosen as the starting value for most of the SMB processes. In section 4.3 , we demonstrate the application of CPM for another system as well as for a wide range of operating parameters.

\subsection{Comparison with other prediction techniques}

In the previous publications, some of the researchers suggested that giving the column certain predetermined initial conditions could speed up the simulation (Hidajat et al., 1986; Minceva et al., 2003). Minceva set the same initial conditions for all the columns, e.g. $c_{\mathrm{A}}=c_{\mathrm{B}}=0.8 \mathrm{~g} / \mathrm{L}$ and $q_{\mathrm{A}}=q_{\mathrm{B}}=2.45 \mathrm{~g} / \mathrm{L}$, while Hidajat selected only some columns (mainly in zone II and zone III) be in equilibrium with half of feed concentration. In our case, the concentrations in fluid phase were set to be $c_{\mathrm{A}}=1.45 \mathrm{~g} / \mathrm{L}$ for columns $3-5$ and $c_{\mathrm{B}}=1.45 \mathrm{~g} / \mathrm{L}$ for columns $4-6$. Correspondingly, the concentrations in solid phase are in equilibrium with fluid phase, $q_{\mathrm{A}}=5.15 \mathrm{~g} / \mathrm{L}$ and $q_{\mathrm{B}}=0$ for column 3, $q_{\mathrm{A}}=4.91 \mathrm{~g} / \mathrm{L}$ and $q_{\mathrm{B}}=3.52 \mathrm{~g} / \mathrm{L}$ for column $4-5, q_{\mathrm{A}}=0$ and $q_{\mathrm{B}}=3.78 \mathrm{~g} / \mathrm{L}$ for column 6 . Both the two scenarios were run in this work and the results are listed in Table 2. The iteration numbers for achieving CSS in the two methods are 161 and 153 respectively, close to or even worse than the value from void column. This indicates that the accelerating simulation by setting initial conditions is limited.

In the previous study, we used "quasi-envelope" method for fast calculation of CSS in SMB (Yao et al., 2010). For comparison, the quasi-envelope method is also used on the enantioseparation process 
of $1,1^{\prime}$-bi-2-naphtol. With the constant integration stepsize ( 8 switches), the iterations needed for achieving CSS is 110 (Table 2). Compared with CMSS, 30\% of computational time is saved. In CPM, with the optimum $\theta$, if taking CMSS as the reference too, the computational efficiency is improved by $59 \%$, much higher than the quasi-envelope method.

Table 2 Iterations required achieving CSS by simulation start with different initial conditions

\begin{tabular}{lc}
\hline Initial conditions & Iterations required to CSS \\
\hline Void column (CMSS) & 156 \\
$c_{\mathrm{A} / \mathrm{B}}=0.8 \mathrm{~g} / \mathrm{L}$ and $q_{\mathrm{A} / \mathrm{B}}=2.45 \mathrm{~g} / \mathrm{L}$ & 161 \\
$c_{\mathrm{A}}=1.45 \mathrm{~g} / \mathrm{L}$ for columns $3-5$ and $c_{\mathrm{B}}=1.45 \mathrm{~g} / \mathrm{L}$ for columns $4-6$, & 153 \\
solid phase in equilibrium with fluid phase & 110 \\
Void column (quasi-envelope method) & \\
\hline
\end{tabular}

\subsection{CPM for sugar separation process under different operating conditions}

The method developed with suggested $\theta$ is applied to another SMB system, the separation of fructose and glucose (Beste et al., 2000). The operation conditions and model parameters are listed in Table 3.

In this process, it takes 159 switches to achieve CSS via successive substitution. Using CPM with $\theta=0.5$, the CSS is obtained after 73 iterations, saving $54 \%$ CPU time relative to CMSS, similar to that of the enantioseparation process discussed above.

Table 3 Parameters for fructose-glucose separation process

\begin{tabular}{ll}
\hline Items & Values \\
\hline Configuration & $2 / 2 / 2 / 2$ \\
Column & i.d. $2.6 \mathrm{~cm} \times 52.07 \mathrm{~cm}$ \\
Porosity & $\varepsilon_{\mathrm{b}}=0.41$ \\
Dispersion coefficient, $\mathrm{cm}^{2} / \mathrm{min}^{-1}$ & $D_{\mathrm{a}}=0.153 \mathrm{v}$ \\
Mass transfer coefficient, $\mathrm{min}^{-1}$ & $k_{\mathrm{e}, \mathrm{A}}=0.72, k_{\mathrm{e}, \mathrm{B}}=0.9$ \\
Feed concentration, $\mathrm{g} / \mathrm{L}$ & $c_{\mathrm{A}}=363, c_{\mathrm{B}}=322$ \\
Switching time, min & $t_{\mathrm{s}}=16.39$ \\
Flow rate, $\mathrm{mL} / \mathrm{min}$ & $Q_{\mathrm{I}}=15.89, Q_{\mathrm{II}}=11.0, Q_{\mathrm{III}}=12.67, Q_{\mathrm{IV}}=9.1$ \\
& $q_{\mathrm{A}}^{*}=0.675 c_{\mathrm{A}}$ \\
Equilibrium isotherm & $q_{\mathrm{B}}^{*}=0.32 c_{\mathrm{B}}+0.000457 c_{\mathrm{A}} c_{\mathrm{B}}$ \\
\hline
\end{tabular}


To verify the adaptability of $\theta$ value at different operation conditions, the SMB processes are modeled at different flow rates, feed concentrations, and switch times, listed in Table 4 (the parameters not shown are kept the same as in Table 3). For comparison, both CMSS and CPM $(\theta=0.5)$ were tried and the corresponding iteration numbers for achieving CSS were obtained.

Table 4 Comparison of computational efficiency by CMSS and CPM at different operation conditions

\begin{tabular}{|c|c|c|c|}
\hline \multirow{2}{*}{ Case } & \multirow{2}{*}{ Operation conditions } & \multicolumn{2}{|c|}{ Iteration number } \\
\hline & & CMSS & $\mathrm{CPM}(\theta=0.5)$ \\
\hline \multirow[t]{2}{*}{ Original } & Flow rate in four zones $(\mathrm{mL} / \mathrm{min}): 15.89,11.0,12.67,9.1$ & 159 & 73 \\
\hline & $c_{\mathrm{A}}=363 \mathrm{~g} / \mathrm{L}, c_{\mathrm{B}}=322 \mathrm{~g} / \mathrm{L}, t_{\mathrm{s}}=16.39 \mathrm{~min}$ & & \\
\hline \multirow[t]{2}{*}{1} & Flow rate in four zones changed to $(\mathrm{mL} / \mathrm{min}): 14,11,12,8$ & 185 & 87 \\
\hline & Other conditions are same as original & & \\
\hline \multirow[t]{2}{*}{2} & Feed concentration changed to $(\mathrm{g} / \mathrm{L}): c_{\mathrm{A}}=400, c_{\mathrm{B}}=350$ & 158 & 73 \\
\hline & Other conditions are same as original & & \\
\hline \multirow[t]{2}{*}{3} & Switch time changed to $(\min ): t_{\mathrm{s}}=18$ & 184 & 85 \\
\hline & Other conditions are same as original & & \\
\hline \multirow[t]{2}{*}{4} & Flow rate in four zones $(\mathrm{mL} / \mathrm{min}): 14,11,12,8$ & 179 & 82 \\
\hline & $c_{\mathrm{A}}=400 \mathrm{~g} / \mathrm{L}, c_{\mathrm{B}}=350 \mathrm{~g} / \mathrm{L}, t_{\mathrm{s}}=18 \mathrm{~min}$ & & \\
\hline \multirow[t]{2}{*}{5} & Flow rate in four zones $(\mathrm{mL} / \mathrm{min}): 18,14,15,10$ & 161 & 74 \\
\hline & $c_{\mathrm{A}}=350 \mathrm{~g} / \mathrm{L}, c_{\mathrm{B}}=350 \mathrm{~g} / \mathrm{L}, t_{\mathrm{s}}=14 \min$ & & \\
\hline
\end{tabular}

It can be seen from Table 4, under different operation conditions, the actual switches required to achieve CSS vary, but the effect on the performance of CPM is trivial. Overall, CPM with $\theta=0.5$ can save the computational time by $53-54 \%$ comparing to CMSS. In other words, the same $\theta$ value is applicable for a certain SMB separation system with diverse operation conditions.

In order to check if the optimum accelerating factor is sensitive to systems, the value of $\theta$ is also optimized for the sugar separation process, shown in Fig.8. It is found the optimum $\theta$ is 0.55 for this SMB system, with an iteration number of 59 for achieving CSS. This proves that the optimum value of $\theta$ do change with a different separation system. So for a new SMB system modeled by CPM, it is beneficial to optimize $\theta$. On the other hand, given that the optimization of $\theta$ is a time-consuming work and the optimum here is also close to $0.5, \theta=0.5$ can also be used as the starting value.

Recently, Lim demonstrated that in the presence of non-homogeneous bed-tails, the mean concentrations no longer reach a steady-state (Lim, 2012). In this case, the CPM method may be limited. 


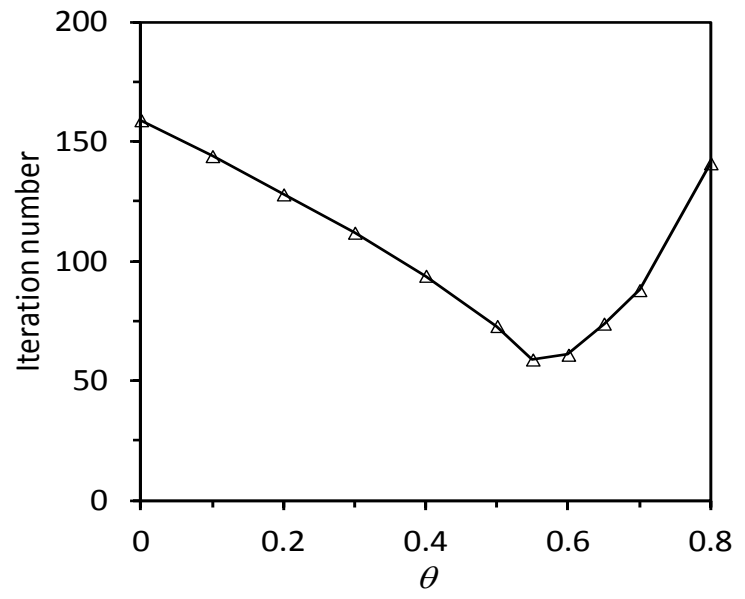

Fig.8. Iteration numbers needed for achieving CSS in SMB process for separation of fructose and glucose by CPM at different $\theta$ values.

\section{Conclusions}

A continuous predicting method is developed to accelerate the calculation of cyclic steady state in SMB process. The key optional variable in this method, the acceleration factor is optimized in the SMB process for enantioseparation of racemic 1,1'-bi-2-naphthol, with maximally $59 \%$ of computational time saved compared to successive substitution. In addition, CPM is validated by another SMB process for separation of fructose and glucose. In diverse operation conditions explored here, without exceptions, the CPM saves $53-54 \%$ of computation time relative to successive substitution.

The method is not only efficient but also facile to implement based on the program of conventional method. Therefore, it could provide a useful numerical tool for simulation and optimization of SMB processes. Besides, the application of this method may not be limited in SMB processes, but could probably also be applicable for other cyclic processes.

\section{Acknowledgements}

This work was supported by the National Natural Science Foundation of China (No.20876128 and No.21206118) and the Fundamental Research Funds for the Central Universities (No.2010121049). 


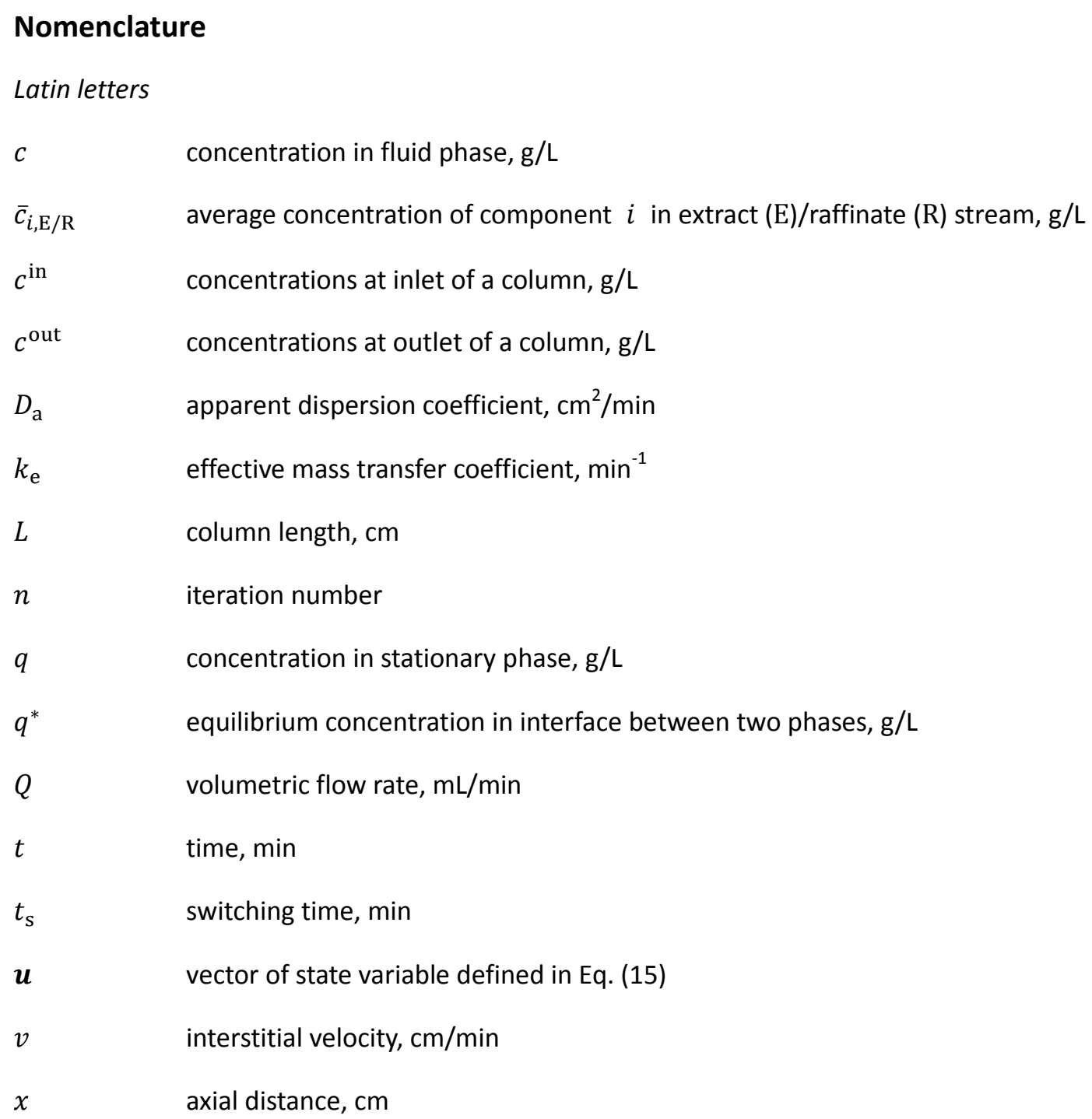




$\begin{array}{ll}\text { Subscripts } & \\ \text { CSS } & \text { cyclic steady state } \\ \text { D } & \text { desorbent } \\ \text { E } & \text { extract } \\ \text { F } & \text { feed } \\ \text { I, II, III, IV } & \text { zone I, zone II, zone III, zone IV } \\ i & \text { component index, } i=A \text { or } B \\ \text { R } & \text { raffinate }\end{array}$

\section{References}

Andersson J, Sahoo D, Mattiasson B. Isolation of potato proteins using simulated moving bed technology. Biotechnol Bioeng 2008; 101: 1256-63.

Beste YA, Lisso M, Wozny G, Arlt W. Optimization of simulated moving bed plants with low efficient stationary phases: separation of fructose and glucose. J Chromatogr A 2000; 868: 169-88.

Biegler LT, Jiang L, Fox VG. Recent advances in simulation and optimal design of pressure swing adsorption systems. Sep Purif Technol 2004; 33: 1-39.

Broughton DB. Production-scale adsorptive separations of liquid mixtures by simulated moving-bed technology, Separ Sci Technol 1984; 19: 723-36.

Ching $\mathrm{CB}$, Ruthven DM. An experimental study of a simulated counter-current adsorption system-I. isothermal steady state operation. Chem Eng Sci 1985; 40: 877-85.

Dunnebier G, Fricke J, Klatt KU. Optimal design and operation of simulated moving bed chromatographic reactors. Ind Eng Chem Res 2000; 39: 2290-304.

Dunnebier G, Klatt KU. Modelling and simulation of nonlinear chromatographic separation processes: a comparison of different modelling approaches. Chem Eng Sci 2000; 55: 373-80.

Gomes PS, Rodrigues AE. Simulated moving bed chromatography: from concept to proof-of-concept. Chem Eng Technol 2012; 35: 17-34.

Gueorguieva L, Palani S, Rinas U, Jayaraman G, Seidel-Morgenstern A. Recombinant protein purification using gradient assisted simulated moving bed hydrophobic interaction 
chromatography. Part II: Process design and experimental validation. J Chromatogr A 2011; 1218: 6402-11.

Guiochon G. Review: preparative liquid chromatography. J Chromatogr A 2002; 965: 129-61.

Hidajat K, Ching CB, Ruthven DM. Numerical simulation of a semi-continuous counter-current adsorption unit for fructose-glucose separation. Chem Eng J 1986; 33: B55-61.

Horneman DA, Ottens M, Keurentjes JTF, van der Wielen LAM. Surfactant-aided size-exclusion chromatography for the purification of immunoglobulin G. J Chromatogr A 2007; 1157: 237-45.

Li P, Xiu G, Rodrigues AE. Proteins separation and purification by salt gradient ion-exchange SMB. AIChE J 2007; 53: 2419-31.

Lim YI. Optimal flushing flow rates in para-xylene simulated moving-bed considering geometric factor of dead volume. Adsorption 2012; 18: 469-82.

Lim YI, Chang SC, Jorgensen SB. A novel partial differential algebraic equation (PADE) solver: iterative space-time conservation element/solution element (CE/SE) method. Comput Chem Eng 2004; 28: $1309-24$.

Lim $\mathrm{YI}$, Jorgensen SB. A fast and accurate numerical method for solving simulated moving bed (SMB) chromatographic separation problems. Chem Eng Sci 2004; 59: 1931-47.

Lubke R, Seidel-Morgenstern A, Tobiska L. Numerical method for accelerated calculation of cyclic steady state of ModiCon-SMB-processes. Comput Chem Eng 2007; 31: 258-67.

Minceva M, Pais LS, Rodrigues AE. Cyclic steady state of simulated moving bed processes for enantiomers separation. Chem Eng Process, 2003; 42: 93-104.

Negawa M, Shoji F. Optical resolution by simulated moving-bed adsorption technology. J Chromatogr 1992; 590: 113-7.

Pais LS, Loureiro JM, Rodrigues AE. Modeling strategies for enantiomers separation by SMB chromatography. AIChE J 1998; 44: 561-9.

Rajendran A, Paredes G, Mazzotti M. Simulated moving bed chromatography for the separation of enantiomers. J Chromatogr A 2009; 1216: 709-38.

Yao HM, Tade MO, Tian YC. Accelerated computation of cyclic steady state for simulated-moving-bed 
processes. Chem Eng Sci 2010; 65: 1694-704.

Yao H, Tian YC, Tade MO. Using wavelets for solving SMB separation process models. Ind Eng Chem Res 2008; 47: 5585-93.

Zhong G, Guiochon G. Analytical solution for the linear ideal model of simulated moving bed chromatography. Chem Eng Sci 1996; 51: 4307-19. 